\title{
Mechanisms of cardiovascular disease in obstructive sleep apnoea
}

\author{
Silke Ryan ${ }^{1,2}$ \\ ${ }^{1}$ Pulmonary and Sleep Disorders Unit, St. Vincent's University Hospital, Dublin, Ireland; ${ }^{2}$ School of Medicine, University College Dublin, Dublin, \\ Ireland \\ Correspondence to: Dr. Silke Ryan, MD, PhD. Department of Respiratory Medicine, St. Vincent's University Hospital, Elm Park, Dublin, Ireland. \\ Email: silke.ryan@ucd.ie.
}

\begin{abstract}
Obstructive sleep apnoea (OSA) is recognized as a major public health burden conveying a significant risk of cardiovascular diseases (CVD) and mortality. Continuous positive airway pressure (CPAP) is the treatment of choice for the majority of patients with OSA but the benefit of CPAP on CVD is uncertain. Thus, a greater understanding of the mechanisms by which OSA leads to CVD might identify novel therapeutic approaches. Intermittent hypoxia (IH), a hallmark feature of OSA, plays a key role in the pathogenesis and experimental studies using animal and cell culture studies suggest that IH mediates CVD through activation of multiple mechanistic pathways such as sympathetic excitation, inflammation, oxidative stress or metabolic dysregulation. Recurrent arousals, intrathoracic pressure swings and concomitant obesity likely play important additive roles in this process. In this review, the available evidence of the pathophysiological mechanisms of CVD in OSA is explored with a specific emphasis on IH, recurrent arousals and intrathoracic pressure swings as the main pathophysiological triggers.
\end{abstract}

Keywords: Obstructive sleep apnoea (OSA); cardiovascular disease; intermittent hypoxia (IH); sleep fragmentation

Submitted Mar 26, 2018. Accepted for publication Jul 27, 2018.

doi: $10.21037 /$ jtd.2018.08.56

View this article at: http://dx.doi.org/10.21037/jtd.2018.08.56

\section{Introduction}

Obstructive sleep apnoea (OSA) characterized by repeated pharyngeal collapse during sleep leading to intermittent hypoxia (IH), sleep fragmentation and excessive daytime sleepiness represents an increasing public health burden. It is a common disorder affecting about $14 \%$ of men and $5 \%$ of women and its prevalence is rapidly rising due to the strong association of OSA with obesity with at least $60 \%$ of OSA subjects being obese $(1,2)$. The major health burden in OSA patients is due to the significant association with cardiovascular and metabolic diseases, such as systemic arterial hypertension, coronary artery disease, heart failure, stroke or type 2 diabetes (T2D) leading to substantial morbidity and mortality and there is continuously growing evidence that this association is, at least in part, independent of the presence of obesity and other common co-morbidities (3-6). The identification of the mechanisms underlying cardiovascular disease (CVD) processes in
OSA is of major importance and despite significant efforts being made, the process remains incompletely understood. Clinical studies using OSA populations have often been limited by inadequate control for frequent co-morbid conditions, in particular obesity, pre-existing cardiovascular disease or medications. Moreover, OSA is a complex and heterogeneous disease and differences in morphology and length of the nocturnal airflow cessations and associated oxygen desaturations, which are not captured by the apnoea/hypopnoea index (AHI) as the traditional OSA severity marker, and also differences in duration of the disease prior to diagnosis are major contributors for the large variability seen between studies results including differences in response to treatment with continuous positive airway pressure (CPAP). Much of our knowledge has been conducted by translational studies using cell culture and animal models. These models allow the usage of genetically homogenous populations and studying various components of the disease in isolation. Furthermore, they 

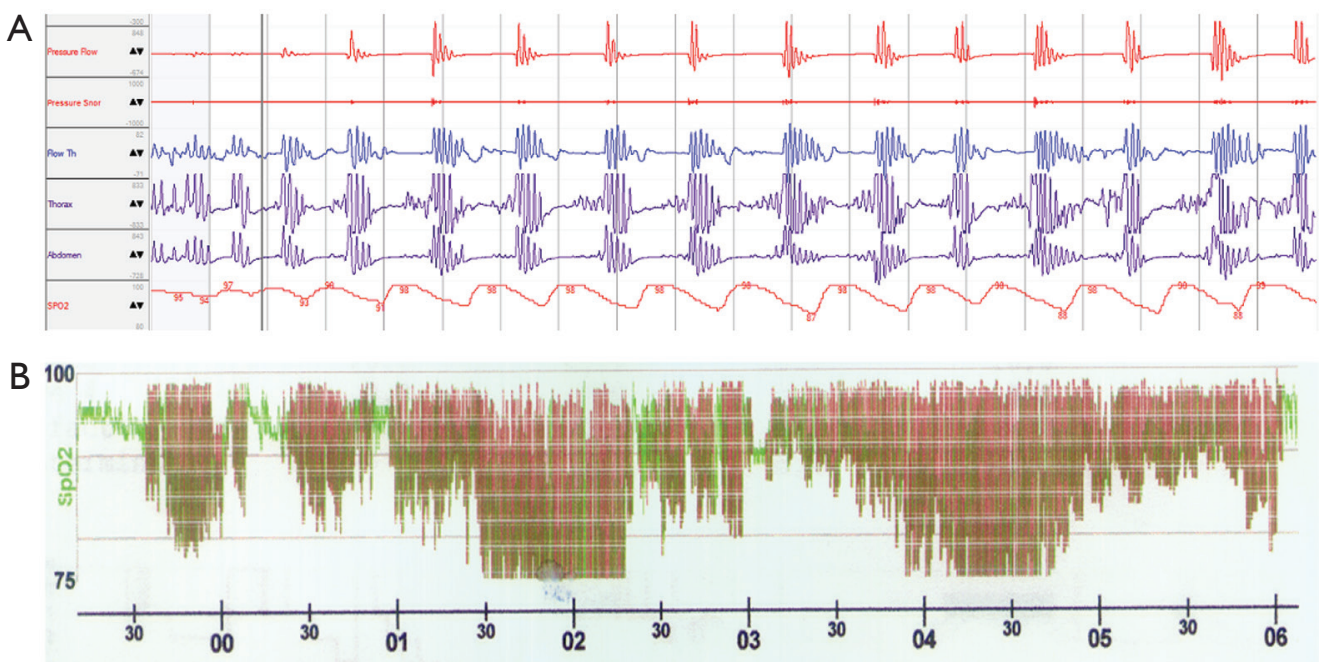

Figure 1 Intermittent hypoxia in OSA. (A) Polysomnographic example of repetitive oxygen desaturation caused by obstructive apnoeas; (B) overnight oximetry in a patient with severe OSA. OSA, obstructive sleep apnoea.

enable precisely controlling the triggering events, both in severity and duration, and thus, have overcome some of the hurdles associated with clinical investigations.

OSA comprises various pathophysiological triggers for CVD, most relevantly IH, recurrent arousals or intrathoracic pressure swings which activate a broad spectrum of pathophysiological pathways including among others sympathetic activation, inflammation, oxidative stress and metabolic dysfunction which in concert lead to cardiometabolic perturbations and subsequent established CVD.

This review will critically discuss our current concept of the pathophysiology of CVD in OSA focusing on the main triggering factors and the intermediate mechanistic pathways. Furthermore, potential future directions for translational research on this subject are provided.

\section{Pathophysiological triggers for CVD in OSA}

\section{IH}

$\mathrm{IH}$ is a hallmark feature of OSA and substantial evidence points to a central role of this factor in the pathophysiology of cardiometabolic diseases. The typical pattern of $\mathrm{IH}$ in OSA with repetitive short cycles of desaturation followed by rapid reoxygenation (Figure 1) bears striking differences to chronic sustained hypoxia observed in other chronic respiratory or cardiac conditions and hence, not surprisingly, leads to considerably different pathophysiological responses. Notably, IH itself is considered as a "double-edged sword" and there is increasing evidence that short exposures to mild IH may lead to adaptive responses through preconditioning effects and hence, may be cardioprotective for patients with mild OSA $(7,8)$. For the purpose of this review, however, the focus lies on the IH pattern typically seen in moderate to severe OSA with frequent short cycles of IH with deeper desaturations and prolonged exposure which leads to numerous deleterious responses. In support of a critical role of such $\mathrm{IH}$ in cardiometabolic processes, various clinical studies have identified the superiority of markers defining the severity of IH in the prediction of cardiovascular outcomes over the traditional AHI which predominantly reflects airflow limitations (9-11). However, the principal evidence of the effects of IH has been provided by experimental models using animals, cell cultures or healthy volunteers. Most of the understanding of $\mathrm{IH}$-induced cardiovascular consequences has arisen from animal studies. The classical non-invasive rodent model of $\mathrm{IH}$ involves housing the animals in specific closed chambers where the animals alternately breathe nitrogen-enriched air to simulate hypoxia and air or oxygen for the reoxygenation phase. Since the first description in the early 1990s (12), this model has been widely used to study consequences of OSA. The IH protocol is usually administered for 
8-12 hours during the light or sleeping phase of the rodents. However, rodents do not sleep continuously as humans do and hence, a significant proportion of the IH occurs while the animal is awake which represents an important limitation. Computer-controlled feedback systems have been developed to trigger the IH during sleep only $(13,14)$, however given the labour- and cost-intensive nature of this approach, most models use IH unlinked to sleep. There is a considerable variation in the duration of the exposure ranging from a few hours to 12 weeks, the frequency of cycles and in the severity of desaturation. Consequently, studies have led to a discrepancy in their results, however, this variability in response to different protocols is in fact reflective of the heterogenous process of OSA and has provided important insight into the differences in outcomes with varying degrees of OSA. Cell culture models of IH have been developed to compliment animal studies and have allowed the investigations of detailed IH-induced cellular responses and signalling mechanisms in different cell lines. These models have continuously advanced over the last years, and we have recently reported a state-ofthe-art system which allows the application of a control exposure in parallel to the IH treatment, the latter closely mimicking the oxygen profile seen in OSA patients, with tight control of other parameters such as temperature or carbon dioxide tension, and thus, this model has overcome many limitations associated with previous descriptions (15). Additional important information has also been provided by human models of IH, particularly gaining insight into responses to short-term IH. Tamisier et al. described a system where healthy volunteers sleep in hypoxic tents with oscillations in oxygen saturation induced by intermittently administering supplemental oxygen via a nasal cannula (16). So far, usage of these models is limited by the cost- and labour-intensive nature of these experiments but will likely increase in the future. However, animal studies will remain indispensable in the field.

Over the last 3 decades, IH models have provided corroborating support of the important role of this feature in the development of numerous CVD in OSA. In rodents, $\mathrm{IH}$ induces a moderate elevation in blood pressure, starting 5 to 8 days after the onset of $\mathrm{IH}$ and usually plateauing even after long exposures of 90 days (12,17-20). The blood pressure elevation persists even after cessation of the stimulus and is independent of the carbon dioxide levels $(12,20,21)$. Importantly, these findings have been reproduced in humans and healthy volunteers exposed to 14 nights of IH experienced an elevation in daytime blood pressure which normalized after 5 days of recovery from the treatment (22). Furthermore, in a cross-sectional analysis of over 11,000 subjects of the European Sleep Apnoea Database (ESADA) cohort, the oxygen desaturation index as principal severity marker of $\mathrm{IH}$ proofed to be significantly more predictive of prevalent hypertension in OSA patients than the AHI (11). Collectively, these data indicate that IH likely contributes to the development of hypertension in OSA.

There is also fast-growing evidence that IH plays an important part in the development of vascular consequences associated with this condition ranging from vascular dysfunction over early atherosclerotic changes to established plaque formation. Treatment of C57BL/6J wild-type mice with $\mathrm{IH}$ for as short as 2 weeks resulted in structural vascular remodelling of the aorta characterized by increased intima-media thickness (23). However, established atherosclerosis in response to $\mathrm{IH}$ in this mouse strain only occurred after long-term (12 week) exposure and in the presence of a high-cholesterol diet, indicating that the IHinduced atherogenic process is amplified in the presence of other risk factors (24). These findings have been reproduced in atherosclerosis-prone apolipoprotein $\mathrm{E}$ deficient $\left(\mathrm{ApoE}^{-/-}\right)$mice with studies demonstrating that $\mathrm{IH}$ accelerates the course of atherosclerosis which is further potentiated with a high-cholesterol diet $(25,26)$. In support of the atherogenic potential of IH, Makarenko et al demonstrated, using a cell-culture approach, a disruption of the endothelial barrier function in response to $\mathrm{IH}$ treatment which was not observed in control cells exposed to alternating cycles of normoxia (27). In keeping with clinical data, there is also substantial evidence that $\mathrm{IH}$ induces vascular functional changes which precede the atherosclerotic process and both, endothelium-dependent impairment of vasodilation and increased vasoconstrictor response have been demonstrated $(19,28,29)$. Endothelin-1 (ET-1) appears to play a major role in this process. IH increases plasma and tissue levels of ET-1 in animals and treatment with the dual endothelin-receptor antagonist bosentan prevents the IH-induced vascular remodelling (30-32). The detrimental cardiovascular effects of chronic IH also extend to the heart. In rats, $\mathrm{IH}$ has been demonstrated to increase the susceptibility to myocardial infarction, to enlarge infarct size and to enhance the 
incidence of ischemic arrhythmias (33-35). Beyond these acute consequences, IH has also shown to lead to cardiac remodelling with biventricular hypertrophy, cardiac fibrosis, dilatation and decreased stroke volume and hence, lending mechanistic insight into the development of heart failure in OSA (28,36-39).

In summary, despite the limitations of the models as describe above, these data provide overwhelming support that IH is likely the most detrimental factor responsible for the cardiovascular pathogenesis in OSA.

\section{Recurrent arousals}

Recurrent arousals are a characteristic feature in OSA leading to sleep fragmentation and subsequent excessive daytime sleepiness as the most debilitating daytime symptom in these patients. Their occurrences depend on the arousal threshold of the individual subject but typically, arousals arise as a result of the interrupted ventilation with subsequent hypoxia, hypercapnia and increased respiratory effort in order to restore ventilation. The arousals are associated with repetitive substantial blood pressure rises as high as $80 \mathrm{mmHg}(40,41)$. However, whether these oscillations in blood pressure contribute to the development of systemic arterial hypertension or vascular disease is still under debate. In a dog model, repetitive upper airway closures during sleep for up to 3 months induced the development of hypertension which however was not seen in response to recurrent arousals induced by acoustic stimuli (20). Similarly, sleep fragmentation through recurrent acoustic stimuli for 35 days did not provoke blood pressure increases in rats (42). However, Launois et al. compared the hemodynamic responses of respiratory and non-respiratory arousals in a porcine model and found, that only respiratory arousals led to blood surges providing a potential explanation to the discrepancy in the result of these studies (43). In addition, long term exposure to sleep fragmentation (12 weeks) induced by intermittent tactile stimulation initiated the development of mild hypertension, endothelial dysfunction and early structural vascular changes in $\mathrm{C} 57 \mathrm{Bl} / 6 \mathrm{~J}$ mice (44). Consequently, recurrent arousals play likely an additive role in the pathophysiology of CVD in OSA, but the detailed contribution of this triggering factor requires further translational studies.

\section{Intrathoracic pressure swings}

Each forced inspiration against the occluded airway during an apnoea episode creates a large negative intrathoracic pressure. These repetitive pressure changes result in elevated venous return with right ventricular overload. Furthermore, the lower intracardiac pressure relative to the extrathoracic structures induces an increase in the afterload of the left ventricle with impaired systolic and diastolic function and chronic stretch of the left atrium (45-47). Simulating OSA by recurrent Mueller manoeuvres in healthy subjects has been used as a model to study the chronic cardiovascular consequences of these pressure swings and studies have shown a potential involvement of this factor in the pathogenesis of aortic dilatation or heart failure (48). Intrathoracic pressure swings seem particularly relevant for the occurrence of atrial fibrillation in OSA. Studies in pigs and humans have suggested that the atrial distension may result in electrophysiological alterations with subsequent induction of atrial premature beats and atrial fibrillation (49,50). Notably, the arrhythmogenic electrophysiological changes in response to intrathoracic pressure swings are not altered by antiarrhythmic drugs such as amiodarone or sotalol explaining, at least in part, the limited efficacy of such medications in patients with OSA $(51,52)$

Taken together, the main characteristic features of OSA-IH, recurrent arousals and intrathoracic pressure swings are all likely contributing to the pathogenesis of CVD. Although described here separately, they occur simultaneously and are closely interrelated. As a net result, they trigger various intermediate pathophysiological pathways which will be reviewed in the following section.

\section{Intermediate mechanisms linking OSA and CVD}

\section{Sympathetic activation}

Several studies have provided evidence of sympathetic excitation in patients with OSA. Three decades ago, Fletcher et al observed increased urinary catecholamine levels in OSA patients in comparison to control subjects and a significant fall following tracheostomy (53). Further studies have since confirmed elevated catecholamine levels both in plasma and urine of OSA patients $(54,55)$ and several randomized-controlled trials have demonstrated a significant fall with CPAP therapy $(56,57)$. Using microneurography as a more direct measurement of sympathetic nervous system activity, Somers et al demonstrated increased muscle sympathetic nervous activity (MSNA) during wakefulness in OSA versus obese 
controls (58). MSNA also correlated with noradrenaline levels (59) and treatment with CPAP therapy significantly lowered MSNA (60).

Good support for the role of sympathetic overactivity in the pathogenesis of hypertension in OSA has been provided by experimental studies and IH and recurrent arousals are likely the principal initiators. In rats, blood pressure increase in response to chronic IH is accompanied by elevation in catecholamine levels $(61,62)$ and moreover, pharmacological and surgical blockage of the sympathetic nervous system abolishes the blood pressure increase $(63,64)$. In an experimental model of healthy humans, 14 nights of IH led to increased MSNA mirroring the blood pressure rise (22). Regarding sleep fragmentation, selective blockage of the cardiac sympathetic innervation abolished increases in heart rate in response to acoustic arousals in a dog model (65). Furthermore, Taylor et al identified in a cohort of otherwise healthy subjects with a wide range of AHI the arousal index as strongest index of daytime muscle sympathetic activity (66).

\section{Inflammation}

The process of atherosclerosis throughout all stages is intimately linked to inflammation (67) and hence, not surprisingly, inflammatory processes are central in the pathogenesis of vascular diseases in OSA. IH has emerged as a potent inflammatory stimulus and thus, plays a major role in this process. Murine studies using $\mathrm{C} 57 \mathrm{Bl} / 6 \mathrm{~J}$ wild-type or $\mathrm{ApoE}^{-/-}$-mice have demonstrated that atherosclerotic changes in response to IH are associated with vascular inflammation with infiltration of pro-inflammatory cells into the vascular wall, increased leucocyte rolling and increased expression of pro-inflammatory cytokines, chemokines and adhesion molecules $(23,25,68)$. Systemic inflammation parallels these changes as evidenced by increased splenocyte proliferation and cytokine expression $(23,25)$. Importantly, normoxic recovery reverses the systemic and vascular inflammation associated with early stages in the atherosclerotic process suggesting a potential therapeutic target (69).

Supported by numerous cell culture and animal studies, activation of nuclear factor-kappa $\mathrm{B}(\mathrm{NF}-\mathrm{\kappa B})$, the master transcriptional regulator of inflammatory responses, is central in the IH-induced inflammation (70-72) and appears to be mediated through the $\mathrm{p} 38$ mitogen-activated protein kinase (MAPK) pathway (73-75). NF- $\mathrm{kB}$ activation has also been demonstrated in harvested endothelial cells from OSA subjects correlating with endothelial dysfunction in these subjects and findings improved with effective CPAP therapy (76). Furthermore, levels of circulating downstream products of NF- $\kappa \mathrm{B}$ activation have been found to be increased in OSA patients versus controls and falling with CPAP therapy supporting the key role of this transcription factor in OSA-associated inflammation (10,77-79). The visceral adipose tissue has emerged as a potential source organ of pro-inflammatory mediators in OSA suggested by studies in vivo and in vitro demonstrating that $\mathrm{IH}$ induces a pro-inflammatory phenotype of the adipose tissue $(15,80-82)$. However, further translational studies are required to determine the detailed role of this organ in vascular inflammation in OSA.

\section{Oxidative stress}

Oxidative stress ensues when the generation of reactive oxygen species (ROS) exceeds the capacity of cellular antioxidant mechanisms to eliminate them. At low or moderate concentrations, ROS play a key role in the regulation of various cell functions and biological processes. At higher levels, however, ROS may lead to oxidative stress and subsequent cell damage $(83,84)$. Substantial evidence arising predominantly from cell culture and animal studies links oxidative stress to the pathogenesis of endothelial dysfunction and atherosclerosis mainly through disruption of the vasoprotective nitric oxide (NO) axis and by mediating vascular inflammation (85).

The contribution of oxidative stress to CVD in OSA has been hypothesised for several years. The repetitive episodes of hypoxia, followed intermittently by rapid reoxygenation could be considered analogous to reperfusion injury which is known to inflict cell damage through ROS production (86). Several animal studies have demonstrated that IH leads to surges of ROS and lipid peroxidation and suggested the contribution of these processes to CVD (87-90). However, the contribution of oxidative stress to cardiovascular perturbations in the human condition of OSA still remains controversial. There is an abundance of cross-sectional studies which have evaluated the association of OSA with oxidative stress markers including among others $\mathrm{F} 2$-isoprostanes, Malondialdehyde (MDA) or oxidized low-density lipoprotein (oxLDL) and have yielded conflicting results $(8,91,92)$. Differences in the subject numbers, matching 
and inclusion criteria have been among the factors responsible for the discrepancy. Furthermore, a recent study suggested an independent relationship between sleep deprivation and oxidative stress markers which may have influenced previous studies (93). Moreover, the effect of CPAP therapy on markers of oxidative stress remains uncertain and several randomized controlled trials failed to show benefit (94-96). However, the reasonably short treatment of up to 8 weeks in most of the studies may have been a limiting factor. Few studies have also evaluated the potential role of antioxidant treatment in OSA with promising results but this subject needs to be explored further using large randomized controlled trials (92).

\section{Metabolic dysregulation}

There is compelling evidence that OSA is associated with metabolic dysfunction and components of the metabolic syndrome, such as insulin resistance (IR), abnormal lipid metabolism or visceral adiposity, and these perpetuations may further contribute to cardiovascular disease processes in OSA. This topic has been subject to detailed reviews $(97,98)$. In brief, there is a particular focus on the relationship between OSA and alterations in glucose metabolism and a large stream of community-based and clinical studies have demonstrated a link of OSA with IR, T2D and glycaemic control. IH plays a key role in the pathogenesis and numerous experimental studies using animal and in vitro models have identified a detrimental effect of IH on glucose metabolism $(15,80,81,99)$. There are several potential mechanisms by which IH mediates its effect on glucose metabolic dysfunction which collectively result in pancreatic $\beta$-cell dysfunction and in IR in the insulin target organs liver, skeletal muscle and adipose tissue $(82,100)$. The latter appears of special importance as IH induces a pro-inflammatory phenotype of the visceral adipose tissue with polarization of adipose tissue macrophages towards an M1 pro-inflammatory subtype, upregulation and secretion of numerous proinflammatory adipokines and subsequent impairment of the insulin-signalling pathway, changes which bear a striking similarity to adipose tissue dysfunction seen in obesity $(15,80,81)$. In addition, sleep fragmentation and sleep deprivation are also linked to metabolic dysfunction and laboratory studies support the assumption that short sleep alters the hormonal regulation of food intake by increasing levels of the appetite-stimulant hormone ghrelin, and by reducing levels of the suppressor leptin, leading to the subjective feeling of hunger with consequent increased caloric intake (101). Furthermore, Gozal et al. revealed a direct effect of sleep fragmentation on adipose tissue inflammation with oxidative stress as the potential mediating mechanism (102).

Similar to cardiovascular diseases (CVD), the benefit of CPAP therapy on metabolic outcomes is still uncertain. Several randomized controlled trials including mainly morbidly obese patients have been performed and have yielded differing results (103-106). However, the methodology of these studies varied significantly in terms of subject numbers, duration of treatment and compliance with CPAP. Treatment seems to be more beneficial to glycemic health in non-obese subjects and improvement in IR with CPAP in obese subjects is unlikely to be expected without concomitant weight loss (94).

\section{Conclusions and future directions}

OSA has evolved as a major public health concern mainly attributed to its significant link with cardiovascular morbidity and mortality. The benefit of CPAP therapy on cardiovascular outcomes remains uncertain. Hence, there is a clear demand of a greater understanding of the detailed pathophysiological mechanisms. Models of OSA characteristics, including animal, cell culture and human models have greatly contributed to our current knowledge and have identified that $\mathrm{IH}$, recurrent arousals and to a lesser extent intrathoracic pressure swings are central in CVD processes through activation of an array of intermediate pathways including sympathetic activation, inflammation, oxidative stress, metabolic dysregulation and mechanical stress (Figure 2). Furthermore, obesity as a frequent concomitant condition is OSA is likely exacerbating the deleterious effects of these triggering factors. However, the relative contribution of the triggering factors, the complex interactions between the different pathophysiological pathways in OSA and the potentially synergistic effects with obesity are still poorly understood. Thus, there is an urgent demand for translational studies which investigate these pathways and factors in combination. Such studies will be critical to identify potential novel therapeutic strategies and to guide the design of future large randomized controlled trials in OSA subjects. 


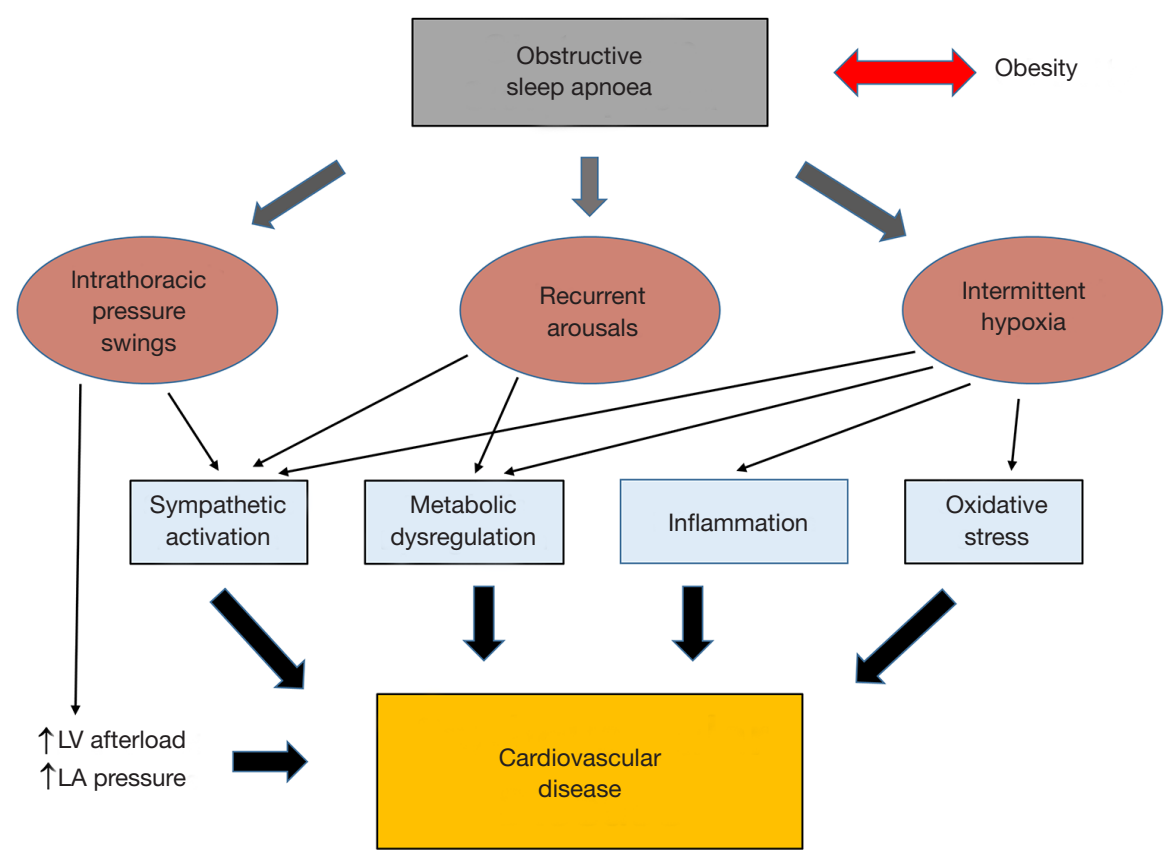

Figure 2 Potential mechanisms of CVD in OSA. CVD, cardiovascular diseases; OSA, obstructive sleep apnoea; LV, left ventricle; LA, left atrium.

\section{Acknowledgements}

Dr. Silke Ryan is supported by an Emerging Investigator Grant from the Health Research Board (HRB) of Ireland.

\section{Footnote}

Conflicts of Interest: The author has no conflicts of interest to declare.

\section{References}

1. Peppard PE, Young T, Barnet JH, et al. Increased Prevalence of Sleep-Disordered Breathing in Adults. Am J Epidemiol 2013;177:1006-14.

2. Peppard PE, Young T, Palta M, et al. Longitudinal study of moderate weight change and sleep-disordered breathing. JAMA 2000;284:3015-21.

3. Kent BD, Grote L, Ryan S, et al. Diabetes mellitus prevalence and control in sleep-disordered breathing: the European Sleep Apnea Cohort (ESADA) study. Chest 2014;146:982-90.

4. Marin JM, Carrizo SJ, Vicente E, et al. Long-term cardiovascular outcomes in men with obstructive sleep apnoea-hypopnoea with or without treatment with continuous positive airway pressure: an observational study. Lancet 2005;365:1046-53.

5. McNicholas WT, Bonsignore MR. Sleep apnoea as an independent risk factor for cardiovascular disease: current evidence, basic mechanisms and research priorities. Eur Respir J 2007;29:156-78.

6. Nieto FJ, Young TB, Lind BK, et al. Association of sleepdisordered breathing, sleep apnea, and hypertension in a large community-based study. Sleep Heart Health Study. JAMA 2000;283:1829-36.

7. Almendros I, Wang Y, Gozal D. The polymorphic and contradictory aspects of intermittent hypoxia. Am J Physiol Lung Cell Mol Physiol 2014;307:L129-40.

8. Lavie L. Oxidative stress in obstructive sleep apnea and intermittent hypoxia--revisited--the bad ugly and good: implications to the heart and brain. Sleep Med Rev 2015;20:27-45.

9. Baguet JP, Hammer L, Levy P, et al. The severity of oxygen desaturation is predictive of carotid wall thickening and plaque occurrence. Chest 2005;128:3407-12.

10. Ryan S, Taylor CT, McNicholas WT. Predictors of elevated nuclear factor-kappaB-dependent genes in obstructive sleep apnea syndrome. Am J Respir Crit Care Med 2006;174:824-30. 
11. Tkacova R, McNicholas WT, Javorsky M, et al. Nocturnal intermittent hypoxia predicts prevalent hypertension in the European Sleep Apnoea Database cohort study. Eur Respir J 2014;44:931-41.

12. Fletcher EC, Lesske J, Qian W, et al. Repetitive, episodic hypoxia causes diurnal elevation of blood pressure in rats. Hypertension 1992;19:555-61.

13. Hamrahi H, Chan B, Horner RL. On-line detection of sleep-wake states and application to produce intermittent hypoxia only in sleep in rats. J Appl Physiol (1985) 2001;90:2130-40.

14. Tagaito Y, Polotsky VY, Campen MJ, et al. A model of sleep-disordered breathing in the C57BL/6J mouse. J Appl Physiol (1985) 2001;91:2758-66.

15. Murphy AM, Thomas A, Crinion SJ, et al. Intermittent hypoxia in obstructive sleep apnoea mediates insulin resistance through adipose tissue inflammation. Eur Respir J 2017;49.

16. Tamisier R, Gilmartin GS, Launois SH, et al. A new model of chronic intermittent hypoxia in humans: effect on ventilation, sleep, and blood pressure. J Appl Physiol (1985) 2009;107:17-24.

17. Lai CJ, Yang CC, Hsu YY, et al. Enhanced sympathetic outflow and decreased baroreflex sensitivity are associated with intermittent hypoxia-induced systemic hypertension in conscious rats. J Appl Physiol (1985) 2006;100:1974-82.

18. Lin M, Liu R, Gozal D, et al. Chronic intermittent hypoxia impairs baroreflex control of heart rate but enhances heart rate responses to vagal efferent stimulation in anesthetized mice. Am J Physiol Heart Circ Physiol 2007;293:H997-1006.

19. Tahawi Z, Orolinova N, Joshua IG, et al. Altered vascular reactivity in arterioles of chronic intermittent hypoxic rats. J Appl Physiol (1985) 2001;90:2007-13; discussion 2000.

20. Brooks D, Horner RL, Kozar LF, et al. Obstructive sleep apnea as a cause of systemic hypertension. Evidence from a canine model. J Clin Invest 1997;99:106-9.

21. Fletcher EC, Bao G, Miller CC, 3rd. Effect of recurrent episodic hypocapnic, eucapnic, and hypercapnic hypoxia on systemic blood pressure. J Appl Physiol (1985) 1995;78:1516-21.

22. Tamisier R, Pepin JL, Remy J, et al. 14 nights of intermittent hypoxia elevate daytime blood pressure and sympathetic activity in healthy humans. Eur Respir J 2011;37:119-28.

23. Arnaud C, Beguin PC, Lantuejoul S, et al. The inflammatory preatherosclerotic remodeling induced by intermittent hypoxia is attenuated by RANTES/CCL5 inhibition. Am J Respir Crit Care Med 2011;184:724-31.

24. Savransky V, Nanayakkara A, Li J, et al. Chronic intermittent hypoxia induces atherosclerosis. Am J Respir Crit Care Med 2007;175:1290-7.

25. Arnaud C, Poulain L, Levy P, et al. Inflammation contributes to the atherogenic role of intermittent hypoxia in apolipoprotein-E knock out mice. Atherosclerosis 2011;219:425-31.

26. Jun J, Reinke C, Bedja D, et al. Effect of intermittent hypoxia on atherosclerosis in apolipoprotein E-deficient mice. Atherosclerosis 2010;209:381-6.

27. Makarenko VV, Usatyuk PV, Yuan G, et al. Intermittent hypoxia-induced endothelial barrier dysfunction requires ROS-dependent MAP kinase activation. Am J Physiol Cell Physiol 2014;306:C745-52.

28. Dematteis M, Julien C, Guillermet C, et al. Intermittent hypoxia induces early functional cardiovascular remodeling in mice. Am J Respir Crit Care Med 2008;177:227-35.

29. Phillips SA, Olson EB, Morgan BJ, et al. Chronic intermittent hypoxia impairs endotheliumdependent dilation in rat cerebral and skeletal muscle resistance arteries. Am J Physiol Heart Circ Physiol 2004;286:H388-93.

30. Briancon-Marjollet A, Monneret D, Henri M, et al. Endothelin regulates intermittent hypoxia-induced lipolytic remodelling of adipose tissue and phosphorylation of hormone-sensitive lipase. J Physiol 2016;594:1727-40.

31. Gras E, Belaidi E, Briancon-Marjollet A, et al. Endothelin-1 mediates intermittent hypoxia-induced inflammatory vascular remodeling through HIF-1 activation. J Appl Physiol (1985) 2016;120:437-43.

32. Lefebvre B, Godin-Ribuot D, Joyeux-Faure M, et al. Functional assessment of vascular reactivity after chronic intermittent hypoxia in the rat. Respir Physiol Neurobiol 2006;150:278-86.

33. Belaidi E, Joyeux-Faure M, Ribuot C, et al. Major role for hypoxia inducible factor- 1 and the endothelin system in promoting myocardial infarction and hypertension in an animal model of obstructive sleep apnea. J Am Coll Cardiol 2009;53:1309-17.

34. Joyeux-Faure M, Stanke-Labesque F, Lefebvre B, et al. Chronic intermittent hypoxia increases infarction in the isolated rat heart. J Appl Physiol (1985) 2005;98:1691-6.

35. Morand J, Arnaud C, Pepin JL, et al. Chronic intermittent hypoxia promotes myocardial ischemia-related ventricular arrhythmias and sudden cardiac death. Sci Rep 2018;8:2997.

36. Campen MJ, Shimoda LA, O'Donnell CP. Acute and 
chronic cardiovascular effects of intermittent hypoxia in C57BL/6J mice. J Appl Physiol (1985) 2005;99:2028-35.

37. Hayashi T, Yoshioka T, Hasegawa K, et al. Inhalation of hydrogen gas attenuates left ventricular remodeling induced by intermittent hypoxia in mice. Am J Physiol Heart Circ Physiol 2011;301:H1062-9.

38. Kato R, Nomura A, Sakamoto A, et al. Hydrogen gas attenuates embryonic gene expression and prevents left ventricular remodeling induced by intermittent hypoxia in cardiomyopathic hamsters. Am J Physiol Heart Circ Physiol 2014;307:H1626-33.

39. Li S, Feng J, Wei S, et al. Delayed neutrophil apoptosis mediates intermittent hypoxia-induced progressive heart failure in pressure-overloaded rats. Sleep Breath 2016;20:95-102.

40. Bangash MF, Xie A, Skatrud JB, et al. Cerebrovascular response to arousal from NREM and REM sleep. Sleep 2008;31:321-7.

41. Morgan BJ, Crabtree DC, Puleo DS, et al. Neurocirculatory consequences of abrupt change in sleep state in humans. J Appl Physiol (1985) 1996;80:1627-36.

42. Bao G, Metreveli N, Fletcher EC. Acute and chronic blood pressure response to recurrent acoustic arousal in rats. Am J Hypertens 1999;12:504-10.

43. Launois SH, Averill N, Abraham JH, et al. Cardiovascular responses to nonrespiratory and respiratory arousals in a porcine model. J Appl Physiol (1985) 2001;90:114-20.

44. Carreras A, Zhang SX, Peris E, et al. Chronic sleep fragmentation induces endothelial dysfunction and structural vascular changes in mice. Sleep 2014;37:1817-24.

45. Kasai T, Bradley TD. Obstructive sleep apnea and heart failure: pathophysiologic and therapeutic implications. J Am Coll Cardiol 2011;57:119-27.

46. Parish JM, Somers VK. Obstructive sleep apnea and cardiovascular disease. Mayo Clin Proc 2004;79:1036-46.

47. Shiomi T, Guilleminault C, Stoohs R, et al. Leftward shift of the interventricular septum and pulsus paradoxus in obstructive sleep apnea syndrome. Chest 1991;100:894-902.

48. Orban M, Bruce CJ, Pressman GS, et al. Dynamic changes of left ventricular performance and left atrial volume induced by the mueller maneuver in healthy young adults and implications for obstructive sleep apnea, atrial fibrillation, and heart failure. Am J Cardiol 2008;102:1557-61.

49. Linz D, Schotten U, Neuberger HR, et al. Negative tracheal pressure during obstructive respiratory events promotes atrial fibrillation by vagal activation. Heart Rhythm 2011;8:1436-43.

50. Schlatzer C, Schwarz EI, Sievi NA, et al. Intrathoracic pressure swings induced by simulated obstructive sleep apnoea promote arrhythmias in paroxysmal atrial fibrillation. Europace 2016;18:64-70.

51. Linz D, Schotten U, Neuberger HR, et al. Combined blockade of early and late activated atrial potassium currents suppresses atrial fibrillation in a pig model of obstructive apnea. Heart Rhythm 2011;8:1933-9.

52. Monahan K, Brewster J, Wang L, et al. Relation of the severity of obstructive sleep apnea in response to antiarrhythmic drugs in patients with atrial fibrillation or atrial flutter. Am J Cardiol 2012;110:369-72.

53. Fletcher EC, Miller J, Schaaf JW, et al. Urinary catecholamines before and after tracheostomy in patients with obstructive sleep apnea and hypertension. Sleep 1987;10:35-44.

54. Dimsdale JE, Coy T, Ziegler MG, et al. The effect of sleep apnea on plasma and urinary catecholamines. Sleep 1995;18:377-81.

55. Elmasry A, Lindberg E, Hedner J, et al. Obstructive sleep apnoea and urine catecholamines in hypertensive males: a population-based study. Eur Respir J 2002;19:511-7.

56. Hedner J, Darpo B, Ejnell H, et al. Reduction in sympathetic activity after long-term CPAP treatment in sleep apnoea: cardiovascular implications. Eur Respir J 1995;8:222-9.

57. Ziegler MG, Mills PJ, Loredo JS, et al. Effect of continuous positive airway pressure and placebo treatment on sympathetic nervous activity in patients with obstructive sleep apnea. Chest 2001;120:887-93.

58. Somers VK, Dyken ME, Clary MP, et al. Sympathetic neural mechanisms in obstructive sleep apnea. J Clin Invest 1995;96:1897-904.

59. Carlson JT, Hedner J, Elam M, et al. Augmented resting sympathetic activity in awake patients with obstructive sleep apnea. Chest 1993;103:1763-8.

60. Narkiewicz K, Kato M, Phillips BG, et al. Nocturnal continuous positive airway pressure decreases daytime sympathetic traffic in obstructive sleep apnea. Circulation 1999;100:2332-5.

61. Dick TE, Hsieh YH, Wang N, et al. Acute intermittent hypoxia increases both phrenic and sympathetic nerve activities in the rat. Exp Physiol 2007;92:87-97.

62. Lesske J, Fletcher EC, Bao G, et al. Hypertension caused by chronic intermittent hypoxia--influence of chemoreceptors and sympathetic nervous system. J 
Hypertens 1997;15:1593-603.

63. Bao G, Metreveli N, Li R, et al. Blood pressure response to chronic episodic hypoxia: role of the sympathetic nervous system. J Appl Physiol (1985) 1997;83:95-101.

64. Fletcher EC, Lesske J, Culman J, et al. Sympathetic denervation blocks blood pressure elevation in episodic hypoxia. Hypertension 1992;20:612-9.

65. Horner RL, Brooks D, Kozar LF, et al. Immediate effects of arousal from sleep on cardiac autonomic outflow in the absence of breathing in dogs. J Appl Physiol (1985) 1995;79:151-62.

66. Taylor KS, Murai H, Millar PJ, et al. Arousal From Sleep and Sympathetic Excitation During Wakefulness. Hypertension 2016;68:1467-74.

67. Libby P. Inflammation in atherosclerosis. Nature 2002;420:868-74.

68. Gileles-Hillel A, Almendros I, Khalyfa A, et al. Early intermittent hypoxia induces proatherogenic changes in aortic wall macrophages in a murine model of obstructive sleep apnea. Am J Respir Crit Care Med 2014;190:958-61.

69. Arnaud C, Beguin P, Levy P, et al. Normoxic Recovery Reverses Intermittent Hypoxia-Induced Systemic and Vascular Inflammation. Chest 2016;150:471-3.

70. Poulain L, Richard V, Levy P, et al. Toll-like receptor-4 mediated inflammation is involved in the cardiometabolic alterations induced by intermittent hypoxia. Mediators Inflamm 2015;2015:620258.

71. Ryan S, Taylor CT, McNicholas WT. Selective activation of inflammatory pathways by intermittent hypoxia in obstructive sleep apnea syndrome. Circulation 2005;112:2660-7.

72. Song D, Fang G, Mao SZ, et al. Selective inhibition of endothelial NF-kappaB signaling attenuates chronic intermittent hypoxia-induced atherosclerosis in mice. Atherosclerosis 2018;270:68-75.

73. Beguin PC, Belaidi E, Godin-Ribuot D, et al. Intermittent hypoxia-induced delayed cardioprotection is mediated by PKC and triggered by p38 MAP kinase and Erk1/2. J Mol Cell Cardiol 2007;42:343-51.

74. Lee MY, Wang Y, Mak JC, et al. Intermittent hypoxia induces NF-kappaB-dependent endothelial activation via adipocyte-derived mediators. Am J Physiol Cell Physiol 2016;310:C446-55.

75. Ryan S, McNicholas WT, Taylor CT. A critical role for p38 map kinase in NF-kappaB signaling during intermittent hypoxia/reoxygenation. Biochem Biophys Res Commun 2007;355:728-33.

76. Jelic S, Padeletti M, Kawut SM, et al. Inflammation, oxidative stress, and repair capacity of the vascular endothelium in obstructive sleep apnea. Circulation 2008;117:2270-8.

77. Dyugovskaya L, Lavie P, Lavie L. Phenotypic and functional characterization of blood gammadelta $T$ cells in sleep apnea. Am J Respir Crit Care Med 2003;168:242-9.

78. Minoguchi K, Tazaki T, Yokoe T, et al. Elevated production of tumor necrosis factor-alpha by monocytes in patients with obstructive sleep apnea syndrome. Chest 2004;126:1473-9.

79. Ryan S, Taylor CT, McNicholas WT. Systemic inflammation: a key factor in the pathogenesis of cardiovascular complications in obstructive sleep apnoea syndrome? Thorax 2009;64:631-6.

80. Carreras A, Zhang SX, Almendros I, et al. Resveratrol attenuates intermittent hypoxia-induced macrophage migration to visceral white adipose tissue and insulin resistance in male mice. Endocrinology 2015;156:437-43.

81. Poulain L, Thomas A, Rieusset J, et al. Visceral white fat remodelling contributes to intermittent hypoxia-induced atherogenesis. Eur Respir J 2014;43:513-22.

82. Ryan S. Adipose tissue inflammation by intermittent hypoxia: mechanistic link between obstructive sleep apnoea and metabolic dysfunction. J Physiol 2017;595:2423-30.

83. Thannickal VJ, Fanburg BL. Reactive oxygen species in cell signaling. Am J Physiol Lung Cell Mol Physiol 2000;279:L1005-28.

84. Hensley K, Robinson KA, Gabbita SP, et al. Reactive oxygen species, cell signaling, and cell injury. Free Radic Biol Med 2000;28:1456-62.

85. Li H, Horke S, Forstermann U. Vascular oxidative stress, nitric oxide and atherosclerosis. Atherosclerosis 2014;237:208-19.

86. Eltzschig HK, Eckle T. Ischemia and reperfusion--from mechanism to translation. Nat Med 2011;17:1391-401.

87. Jun J, Savransky V, Nanayakkara A, et al. Intermittent hypoxia has organ-specific effects on oxidative stress. Am J Physiol Regul Integr Comp Physiol 2008;295:R1274-81.

88. Peng YJ, Nanduri J, Yuan G, et al. NADPH oxidase is required for the sensory plasticity of the carotid body by chronic intermittent hypoxia. J Neurosci 2009;29:4903-10.

89. Troncoso Brindeiro CM, da Silva AQ, Allahdadi KJ, et al. Reactive oxygen species contribute to sleep apnea-induced hypertension in rats. Am J Physiol Heart Circ Physiol 2007;293:H2971-6.

90. Zhan G, Serrano F, Fenik P, et al. NADPH oxidase mediates hypersomnolence and brain oxidative injury in a murine model of sleep apnea. Am J Respir Crit Care Med 
2005;172:921-9.

91. Badran M, Ayas N, Laher I. Cardiovascular complications of sleep apnea: role of oxidative stress. Oxid Med Cell Longev 2014;2014:985258.

92. Lira AB, de Sousa Rodrigues CF. Evaluation of oxidative stress markers in obstructive sleep apnea syndrome and additional antioxidant therapy: a review article. Sleep Breath 2016;20:1155-60.

93. DeMartino T, Ghoul RE, Wang L, et al. Oxidative Stress and Inflammation Differentially Elevated in Objective Versus Habitual Subjective Reduced Sleep Duration in Obstructive Sleep Apnea. Sleep 2016;39:1361-9.

94. Jullian-Desayes I, Joyeux-Faure M, Tamisier R, et al. Impact of obstructive sleep apnea treatment by continuous positive airway pressure on cardiometabolic biomarkers: a systematic review from sham CPAP randomized controlled trials. Sleep Med Rev 2015;21:23-38.

95. Paz YMHL, Hazen SL, Tracy RP, et al. Effect of Continuous Positive Airway Pressure on Cardiovascular Biomarkers: The Sleep Apnea Stress Randomized Controlled Trial. Chest 2016;150:80-90.

96. Sivam S, Witting PK, Hoyos CM, et al. Effects of 8 weeks of CPAP on lipid-based oxidative markers in obstructive sleep apnea: a randomized trial. J Sleep Res 2015;24:339-45.

97. Bonsignore MR, Borel AL, Machan E, et al. Sleep apnoea and metabolic dysfunction. Eur Respir Rev 2013;22:353-64.

98. Kent BD, McNicholas WT, Ryan S. Insulin resistance, glucose intolerance and diabetes mellitus in obstructive sleep apnoea. J Thorac Dis 2015;7:1343-57.

Cite this article as: Ryan S. Mechanisms of cardiovascular disease in obstructive sleep apnoea. J Thorac Dis 2018;10(Suppl 34):S4201-S4211. doi: 10.21037/jtd.2018.08.56
99. Drager LF, Li J, Reinke C, et al. Intermittent hypoxia exacerbates metabolic effects of diet-induced obesity. Obesity (Silver Spring) 2011;19:2167-74.

100.Drager LF, Jun JC, Polotsky VY. Metabolic consequences of intermittent hypoxia: relevance to obstructive sleep apnea. Best Pract Res Clin Endocrinol Metab 2010;24:843-51.

101. Spiegel K, Tasali E, Penev P, et al. Brief communication: Sleep curtailment in healthy young men is associated with decreased leptin levels, elevated ghrelin levels, and increased hunger and appetite. Ann Intern Med 2004;141:846-50.

102. Gozal D, Khalyfa A, Qiao Z, et al. Protein-Tyrosine Phosphatase-1B Mediates Sleep FragmentationInduced Insulin Resistance and Visceral Adipose Tissue Inflammation in Mice. Sleep 2017;40.

103. Hoyos CM, Killick R, Yee BJ, et al. Cardiometabolic changes after continuous positive airway pressure for obstructive sleep apnoea: a randomised sham-controlled study. Thorax 2012;67:1081-9.

104. Lam JC, Lam B, Yao TJ, et al. A randomised controlled trial of nasal continuous positive airway pressure on insulin sensitivity in obstructive sleep apnoea. Eur Respir J 2010;35:138-45.

105. Weinstock TG, Wang X, Rueschman M, et al. A controlled trial of CPAP therapy on metabolic control in individuals with impaired glucose tolerance and sleep apnea. Sleep 2012;35:617-25B.

106. West SD, Nicoll DJ, Wallace TM, et al. Effect of CPAP on insulin resistance and $\mathrm{HbA1c}$ in men with obstructive sleep apnoea and type 2 diabetes. Thorax 2007;62:969-74. 